\title{
MACIV - A MULTI-AGENT SYSTEM FOR RESOURCE MANAGEMENT ON CIVIL CONSTRUCTION COMPANIES
}

\author{
José Manuel Fonseca* \\ jmf@uninova.pt \\ Eugénio de Oliveira ${ }^{*}$ \\ eco@garfield.fe.up.pt
Adolfo Steiger-Garção*
asg@uninova.pt
*FCT/UNL - Faculdade de Ciências e Tecnologia
Universidade Nova de Lisboa
2825 Monte de Caparica - Portugal \\ ${ }^{*}$ FEUP - Faculdade de Engenharia \\ Universidade do Porto \\ Rua dos Bragas - Porto - Portugal
}

\begin{abstract}
Motivated by the interest of a civil construction company and the very attractive scenario characteristics for the application of Distributed Artificial Intelligence techniques, we are developing MACIV, a Multi-Agent System for Resource Management in Civil Construction Companies (CCC). This system is based on Distributed Artificial Intelligence techniques that allow a completely decentralised managing of the different resources on a CCC. In this paper, the general system architecture is presented and explained. In order to achieve good quality management solutions special coalition formation and negotiation techniques were developed and are also presented. In order to enhance the clarity of the presentation an application example is developed and explained in the final section of the paper.
\end{abstract}

Keywords: Automation, Artificial Intelligence, Multi-Agent Systems, Negotiation.

\section{INTRODUCTION}

The increasing business globalisation caused by the easier communications introduced by the Internet is pushing companies to a harder competitiveness in a more exigent global market. In order to survive in this highly competitive scenario the adoption of state of the art information technology is essential and can make the difference. This is true in almost all business sectors and civil construction companies are no exception to this rule.

The correct resource selection and price estimation for each task is certainly a very important part of a managing system for civil construction companies. To achieve such system a centralised or a decentralised solution can be adopted. The centralised solution has the advantage of simplicity and the possibility of easily getting a global view of the problem allowing easier optimisation. The decentralised solution has some drawbacks such as the difficulty on getting a global optimisation and the dependency on communications but it also has important advantages. The possibility of a detailed modelling by representing each physical entity as a software agent, the distribution of the work load for different machines, the failure tolerance by keeping some nodes working even if some others fail and the possibility of locally processing the local events are some of the advantages that can be pointed out to a decentralised solution. This was the approach adopted on the design of MACIV project that aims to the development of a distributed system for resource selection and price estimation for civil construction companies using distributed artificial intelligence techniques. In the last years, Distributed Artificial Intelligence (DAI) techniques have been successfully applied to a large number of different problems. Distributed Vehicle Monitoring [4], Manufacturing [15], Process Control [9], Telecommunications [7], Air Traffic Control [20], Transportation Systems [5], Information Management [11], Electronic Commerce $[18,3,8]$, Business Process Management [10] and many others are examples of well known applications of this kind of techniques. The increasing number of users of the World Wide Web and the tremendous business around it (see $[1,26]$ for more information about Internet statistics) is also 
attracting the attentions for automated negotiation and user assistance systems mainly based on DAI techniques.

Civil construction companies are typically a good and promissory scenario for studying and developing multi-agents systems. In fact, they are usually geographically distributed because all medium and large building companies have many different building, storing and managing places distant from each other. The high number of tasks involved in building and road construction are often easily and almost linearly decomposable. The great diversity of resources gives the possibility of a rich and interesting modelling. The function multiplicity of many of the resources creates different alternatives that are a potential optimisation problem and the high dynamics of this kind of scenarios, with a large number of unpredictable situations due to weather changes, machinery failures, etc, often leads to the necessity of complete replanning. All this factors are challenging for the application of DAI techniques and can constitute an ideal scenario for the demonstration of their validity.

\section{MACIV GENERAL ARCHITECTURE}

The goal of the MACIV Project is the development of a computational system for the resource management on civil construction companies. In order to achieve an adequate solution and take into account the specific characteristics of the problem, it was decided to adopt a decentralised solution based on distributed artificial intelligence techniques. The system's conceptual model is depicted on next figure.

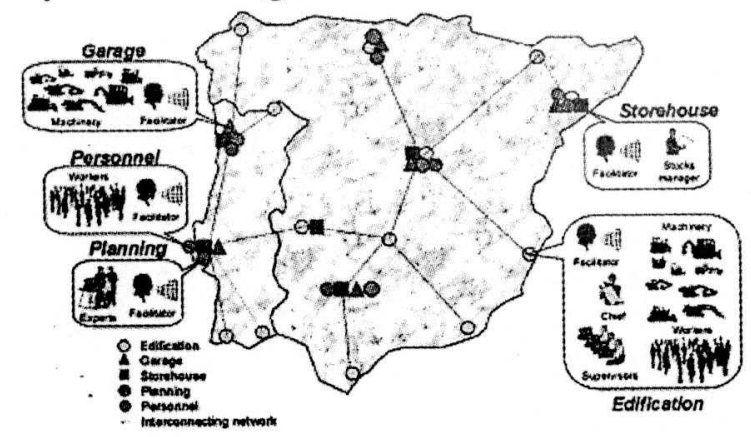

Figure 1-General system architecture.

As it can be seen, the system is hierarchically organised as a collection of macroagents interconnected by communication link that can be a local area network or the Internet. A macro-agent is considered to be a functional grouping of one or more computers interconnected trough a local area network and devoted to local resources managing. Because communication problems are always possible in such a geographically distributed system, the macroagents are prepared to work both on local mode and global mode. When communication problems occur the macroagents automatically enter the local mode that allows the managing of the local resources without any intervention of external entities. When the communication with the rest of the network is re-established the macros-agents return to the global mode participating in the global managing decisions.

A macroagent was defined to be installed at each Edification, Garage (where inactive machinery is stored) and Warehouse (where building materials are stored). There is also one macroagent representing the Planning Department and another representing the Personal Department. The Planning Department is especially important in the system because it will be the main source for new tasks launched in the system. In each macroagent there is a special agent called Facilitator constituting what is usually called a federated system [25, 12]. These agents have two main functions: address servers and communication switches. In order to be known in the system, all agents must register themselves on their local facilitator at startup. The facilitator will then provide the service of giving communication address by name and vice-versa. However, they are specially useful as communication switches. The startup registration of each agent must contain the specification of the capabilities of each agent in order to allow the facilitators to build a database containing the capabilities of all the agents placed at their macroagent. When a new announcement is to be launched in the system it can be directly addressed to some specific agents or broadcasted to all the Facilitators that will send it just to the potentially interested agents. This will save a large number of unnecessary messages when compared to the simpler solution of broadcasting the announcements to all the agents without any criteria.

Another important design decision that must be pointed out here is the fine grain modelling adopted in the design of the system. Due to the great resource diversity and the frequent differences between entities belonging to the same class, our choice was the definition of a software agent to computationally represent each physical entity. This solution allows a very detailed modelling by specifying the particular characteristics of each entity on its software representative.

Each macroagent has specific functions accordingly to the type of installation it represents. The macroagent Edification represents an active working place. It includes the Stocks Manager agent that manages the stocks of materials existent in the place, the supervisors that will give feedback to the system about the performance of each physical entity, the chief that represents the local responsible and the machinery and the workers representative agents. The macroagent Planning Department is responsible 
for the creation and launching of new tasks in the system. It will use the system both to obtain information about availability and/or cost of machinery and workers in order to produce adequate estimations for new edifications and to select the executors for new tasks. The macroagent garage represents the places were inactive machinery is stored. It is a very simple macroagent that contains just a Facilitator and the agents representing the inactive machines. Similarly to the Garage, the Warehouse macroagent represents the places were building materials are stoked. Instead of the agents representing the inactive machines this macroagent contains a database containing the stocks at each site. The macroagent personal is used to receive the inactive workers software representatives. Always that any any worker does not have any task to perform his software representative will move to this macroagent in order to allow a consistent geographical location. It will move to a new location as soon as it gets a new occupation.

\section{RESOURCE SELECTION}

The selection of the adequate resources to execute each task launched on the system is a core part of the MACIV project. The negotiation protocol proposed is inspired on the Contract Net Protocol (CNP) originally proposed by Randall Davis [2]. The CNP protocol is based on a announcement, bid and selection process that has been progressively extended to include bounded rationality [24], decentralised task decomposition [19], commitment policies [22], temporal restrictions [17], coalition formation [14], future commitments [23] between many others. However, safe and adequate protocols for agents coalition formation, based on inter (between coalitions) and intra coalition (inside coalitions) negotiation are still an open question.

Our work is specially dedicated to the task distribution problem whenever it is possible that a task is too large to be executed by a single agent and must be executed by a team of agents grouped in what we call a coalition. Inter-coalition negotiation involves the dynamic adaptation of the coalitions initial propositions to new values in order to adapt their own proposals to the solutions (working prices) proposed by the other coalitions. The changes in the coalitions proposed values have as a consequence changes on the prices of some (or all) of their elements. This implies the need for a process of negotiation also inside the coalition (intra-coalition negotiation).

In the task distribution problem the agents usually try to solve the global problem with the minimum involvement of their owns. In the MACIV system all agents should pay their existence by working and getting profits. This strategy implies that they always try to maximise their occupation trough the execution of all possible tasks at the highest possible price. The detailed model of each physical entity (machine or worker) by means of a software agent that contains the knowledge about all its relevant characteristics allows the system to find out a very precise decision about the best resource and the most adequate price for each task execution. Through the introduction of learning techniques, the system will also be able to adapt itself to the changes on the agents characteristics as well as to give important information about the agents long term abilities and usefulness. This is an ongoing project which includes the end-user company who is giving to the development team an important expertise about the problem.

In this paper we present a multi-stage algorithm for negotiation that allows agents coalition formation and inter as well as intra coalition negotiation in order to achieve a final solution which reflects the economical conditions of the society of agents in which it is launched.

\section{COSTS CALCULATION}

Due to the high cost of many of the resources owned by typical CCC, price calculation is an important part any resource managing system. Accordingly to the Net Present Value (NPV) criterion [Pin95], a resource is considered worthwhile, if the present value of the expected cashflows in the future is higher than it's actual cost:

$$
N P V=-C+\sum_{i=1}^{N} \frac{\pi_{i}}{(1+R)^{\gamma}}+\frac{V_{r}}{(1+R)^{v}}>0
$$

where $C$ is the amount of investment, $R$ the discount rate that is used to discount the future stream of profits (it can be a market interest rate or some other rate), $N$ the number of years that we expect the investment to generate profits, $\pi_{i}$ the profit of year $i$ and $V_{r}$ the residual value of the equipment after $N$ years of usage. In order to simplify the previous equation, we can consider the annual profits constant along the life time of the investment $\left(\forall_{i \in[1 . N]} \pi_{i}=\pi\right)$. Using this simplification the minimum value for the annual incoming will be:

$$
\pi>R \frac{C(1+R)^{v}-V_{r}}{(1+R)^{v}-1}
$$

For this analysis the inflation and the risk of the investment have also to be taken into account. Inflation is automatically considered provided that the annual cash flow $\pi$ and the discount rate $R$ are both nominal or real values ${ }^{1}$. The risk of investment is usually considered by the increase of the discount rate by adding a risk premium to the risk-free rate $R$.

\footnotetext{
1 The real interest rate is the nominal interest rate minus the rate of inflation [Sam92].
} 
Therefore, the previuos equation can be maintained unchanged.

In order to guarantee any machine a minimum annual cash flow of $\pi$, a daily fixed cost for each resource in the company, independent of its activity, and equal to the value $\pi$ divided by the number of usual working days in the year must be introduced. Therefore, the accounting cost for the activity of any resource will be calculated as:

$$
A C=F C+V C+P
$$

where $A C$ is the accounting cost, $F C$ the fixed cost, $V C$ the variable cost of the resource and $P$ the expected profits from that activity. Variable costs are the costs in consequence of the activity - fuel consumption, tyres degradation, etc. The profits $P$ are the way for the resources to compensate the loses caused by inactivity periods due to maintenance programs or unemployment.

Although the accounting cost is correct from the accountant point of view, the economist and managers must have a forward-looking concerning the firm. They also should be concerned about the opportunity cost, the cost associated with opportunities that are foregone by not putting the firm's resources to their highest value use [Pin95]. Therefore, when evaluating what we call the economical cost - EC - of performing any activity, we must also consider the two possible alternatives to the present activity: the unemployment with a probability of $\mathrm{p}_{u}$ (where it will get a loss of $F C+P$ ) or other alternative activity with probability $\left(1-p_{u}\right)$ (where the resource could get an additional profit of $A C-E C)$. Therefore, the equation for the economical cost will come:

$$
E C=A C+\left(1-p_{u}\right)(A C-E C)-p_{u}(F C+P)
$$

Two extreme cases can be pointed out to exemplify the significance of the last equation. Suppose that $\mathrm{p}_{\mathrm{u}}=0$ meaning that the resource can be sure that it will get in the future an alternative task. In this case $E C=F C+V C+P$ what is exactly the accounting cost (see equation 4). On the opposite case, $\mathrm{p}_{\mathrm{u}}=1$ means that there is no probabilistic chance to get any other task than the current one. In this case $E C=V C$ which means that in the costs calculation for employing that particular resource on that task, only the variable costs must be paid. From the economical point of view, a resource can perform any task (it is suitable for) since it is paid higher than its economical cost. This tell us that a resource will have a negotiation margin between its accounting cost, which we can consider an ideal revenue, and its economical cost that can be seen as a minimum allowable value.

Expressing the activity profit as a percentage $p_{p}$ of the total cost, $P=p_{p}(F C+V C)$, equation 4 will became:

$$
E C=\frac{F C\left(2-2 p_{u}+2 p_{p}-2 p_{u} p_{p}\right)+\left(2-p_{u}+2 p_{p}-2 p_{u} p_{p}\right)}{2-p_{u}}
$$

As it can easily be seen, the unemployment probability is fundamental in the economical cost calculation. In the next figure the evolution of $E C$ according to the value of $p_{u}$ for two different agents $A$ and $B$ with $F C=100, V C=150, P=0 \%$ and $F C=300, V C=50, P=0 \%$ respectively is shown.

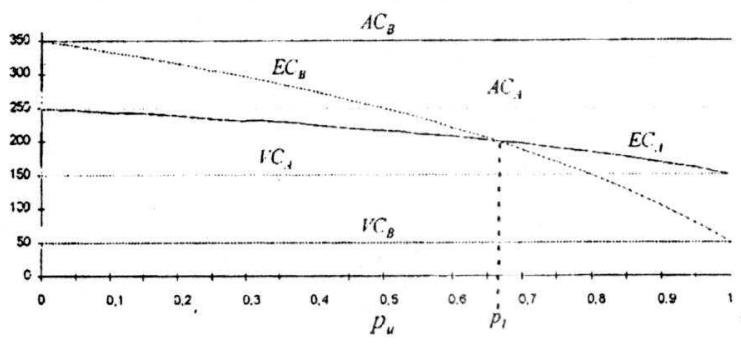

Figure 2 - Economic cost variation with $\mathrm{p}_{\mathrm{u}}$ for two dilferent agents.

To calculate the unemployment probability $p_{\mathcal{u}}$ we propose the following formula:

$$
\left\{\begin{array}{c}
\text { if } N_{\mathrm{c}}-1>N_{\mathrm{a}} \Rightarrow p_{\mathrm{u}}=1-\frac{N_{\mathrm{a}}}{N_{\mathrm{c}}-1} \\
\text { if } \mathrm{N}_{\mathrm{c}}-1 \leq \mathrm{N}_{\mathrm{a}} \Rightarrow \mathrm{p}_{\mathrm{u}}=0
\end{array}\right.
$$

where $N_{a}$ represents the expected number of new announcements and $N_{c}$ the number of agents competing for the announced task. This formula is very easy to interpret. If the number of expected announcements is higher than the number of agents competing for its execution it is expected to have activities for all of them and therefore the unemployment probability is zero. Otherwise, only $N_{a}$ of them will get a task what will lead to the value $I-N_{d}\left(N_{c^{-1}}\right)$. While the number of competitors can be supplied by the announcer, the expected number of announcements may be estimated based on the past activities of the system. For this propose, the last years of activity should be registered and analysed in order to get a correct estimation for that parameter.

We may consider our work near to Sandholm's work [24]. However, some differences can be pointed out: we are not only interested on assembling coalitions of agents for getting the best price but our main concern is first to assemble a good coalition to execute the announced task, an then try to calculate a fair price having also in mind each agent's interests as well as the interest of the multi-agent system (a company). Moreover, we have a significant difference in the way we are calculating coalition bids for an announced task. Once we are proposing a price for a presumable future task execution, we are taking into account the dynamics of the environment like, for instance, future agents' opportunities. This concern leads us to the calculation of an agent's economical cost, where some of these factors are reflected, and not just the marginal costs, as it seems to be the case with Sandholm. 


\section{THE NEGOTIATION PROTOCOL}

We can look at the accounting and economical costs as maximum and minimum acceptable costs for a task execution. In order to adjust the cost accordingly to the company internal market situation, we adopted an iterative algorithm that can be considered as an extension of the well known Contract Net Protocol [2]. This protocol is composed by six successive steps:

- 1st step - announcing - the announcer agent sends the task announcement to all the potentially interested agents.

- 2nd step - task evaluation - the interested agents compute the estimated price for the task execution and sends it out to the announcer.

- 3rd step - selection phase - the announcer collects the bids and rejects those coming from agents that don't fulfil (from his point of view) the basic preconditions for election. The announcer must then calculate all the possible agents coalitions that can solve the problem. Notice that our concept of coalition comes from the functional point of view. At this stage, our main interest is not to maximise the profits of the agents like in $[16,21]$ but the constitution of teams that only together can solve the problem. The individual profits will result from the negotiation process.

The calculation of all the possible teams is a computationally heavy process. However, it can be efficiently computed for a large number of agents. If the number of agents is too high, a simple selection based on the initial cost/performance ratio can be used to dramatically reduce the number of coalitions under consideration (see [13] for a more detailed discussion).

In every approved coalition one of its members is designated as "coordinator" and will be responsible for the intra-coalition negotiation. This election will be done trying to distribute evenly the responsibilities between all the agents in the process. Finally, the announcer must send out to all coordinators the information that they are coordinators of a coalition, the composition of the coalition team, the individual prices proposed by each of the agents in that coalition and the best price achievable at this stage. In the limit, it will also be possible to have "coalitions" of one single agent.

- 4th step - market manipulation - the announcer sends out to the coordinators the best bid achievable at this stage.

- 5th step - price adjusting - the coordinator of each coalition evaluates the possibility to improve the actual coalition offer. For that, he establishes an intra-coalition negotiation with the coalition partners, as we will see later. If it is not possible to obtain a price lower than the currently best one, the agent quits the coalition from the process sending out a message to the announcer communicating this fact. If a lower price is achievable it sends out the new offer to the announcer.

- 6th step - price selection - the announcer, after receiving all the bids from all the coordinators (offering better conditions or quitting), evaluates the best offer.

Steps 4,5 and 6 are repeated until all but one coalition remains in the negotiation. A timeout can also be defined to limit the negotiation process.

The proposed negotiation protocol has however a disadvantage for agents participating in more than one coalition simultaneously. If an agent participates in more than one coalition it can self depreciate its own value by competing with itself in the different coalitions. In fact, if this agent agrees to lower its cost when it is participating on the leading coalition it will be fighting against itself. In order to overcome this problem the "frozen costs" solution was adopted. The frozen costs solution determines that the auctioneer is obliged to communicate to the leading coalition coordinator (or isolated agent) that its proposal is the current best proposal. Then the coordinator divulges this information to all the participants on this coalition that will not agree on lowering their prices until the situation is changed. This protocol guarantees to all the agents that they will not contribute to their own depreciation. It also has the advantage that important resources that have no direct concurrency keep their costs unchanged reflecting their importance to the final solution. Its what we call the "value of being important" [6].

\section{AN APPLICATION EXAMPLE}

In this session an application example that demonstrates how the negotiation algorithm works when applied to a simulated problem is developed. Lets consider that an announcement of $900 \mathrm{~m}^{3} /$ day soil movement during 3 weeks is launched with an antecedence of 10 months.

This announcement is sent to all the facilitators that using their knowledge about the capabilities of their local agents send it just to the potentially interested agents (Figure 3 - Negotiation step 1). Those agents will then computed their initial bid value and their economical cost. The economical cost (that is kept private to each bidder) will be the minimum acceptable value along the negotiation process. For the estimation of the economical cost the unemployment probability $p_{\mathcal{u}}$ should have been calculated based on the historical data accessible by the system. However, the values presented here have been manipulated in order to improve comprehensibility on the example.

Suppose that six agents are available for that date 
with the characteristics presented on Table 1.

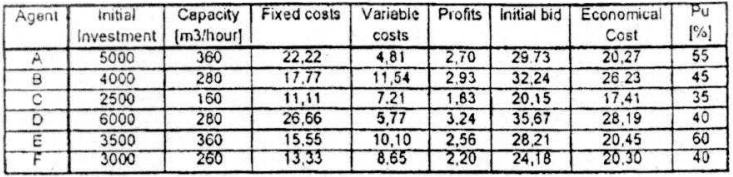

Table 1. Agents economical characteristics.

The estimated costs for this activity, as calculated by each agent, are shown on the column "Initial bid". For the fixed costs calculation a residual value $\left(V_{r}\right)$ of $20 \%$ of the initial investment was used. After receiving the announcement, all the agents answer it with their initial bid value (Figure 3 - Negotiation step 2). As it can be seen in the previous table, none of the agents can actually solve the problem alone because none has enough capacity. On negotiation step 3 the announcer calculates the acceptable coalitions and broadcasts it, together with the value of the best offer received ( 82.12 in this example) to the respective coordinators (shown underlined on the coalition teams). The initial bid of each coalition is considered to be the sum of the individual costs of its agents. Notice that all coalitions containing at least one superfluous element or not powerful enough to solve the problem are rejected. Agents that can solve the problem by themselves are considered coalitions with one single agent (none in this example).

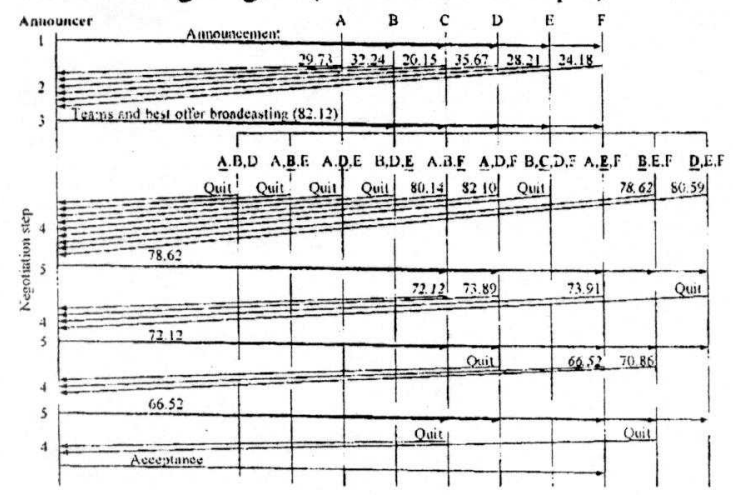

Figure 3 - Negotiation evolution.

After step 3 intra-coalition negotiation starts with the coordinators trying to reduce their own coalitions offers. In this example, the strategy chosen for all the coordinators was to get a new offer $10 \%$ lower than the best offer at each round. From previous figure we can see that five of the ten coalitions quit in the first negotiation round. Looking at the coalition 1 , if we add up the minimum values for agents $A, B$ and $D$ we get 74.70 which is lower than 82.12. However, this coalition quits. This is because agent $\mathrm{A}$ is frozen (it is participating on the leading coalition) and the minimum prices for agents $B$ and $D(26.23+28.19)$ added up to the offer of agent $A$ in the previous round (29.73) is 84.15 which is higher than 82.12 . A similar situation happens with all other coalitions quitting in the first round. In this first round four coalitions make new offers. Notice that the leading coalition in one round doesn't bid in the next one.
The negotiation process keeps going until just one coalition remains and wins the task. In this case coalition 8 wins having a cost of 66.52 . This value is therefore considered to be the minimum acceptable cost for the task execution.

This way, the expert using the system will get the information that any price for the activity going from this minimum value 66.52 to the ideal value 82.12 (or even higher) is worthwhile for the company. Expert final decision must be taken accordingly to his knowledge of the external market and the company strategy. This cost estimation, calculated a long time prior to the task execution, is based on beliefs about the future activity and will be used for the company to bid externally for new tasks. If the task is awarded to the company the resources that presented the best bid (in this example A, E and F) will be reserved for the task execution.

As we previously said, when the execution date arrives, the problem should be revised. The best resources to execute the task can be different from those that presented the best offer in the budget estimation step. This can happen due to two different reasons: the scenery changed (new machines were acquired, machines went out of service, etc) or the agents beliefs have not be confirmed. When we are really close to the execution date, the unemployment probability of disposable resources is around one. Therefore, the economic cost is close to the variable or marginal cost. This means that the final decision must be based on the variable costs. This means that, in this example, if the resources available at the execution date are the same as they were at the first announcement date $(A, B, C, D, E$ and $F$ ) it will be coalition 6 , comprising elements $A, D$ and $F$ to win the re-announcement. This new agents coalition will be chosen to perform the task because its marginal cost $19.2(4.81+5.77+8.65)$ is the best of all produced by other coalitions.

We believe that this is an interesting result because the costs are initially calculated taking into consideration the opportunities of future commitments that, as they are future events, have to be estimated on a probabilistic basis. Of course that the future can not be predicted exactly and therefore, whenever it is possible, revisions must be done to adjust the estimations to reality. This is what is achieved with this costs revision step at the execution task date.

\section{CONCLUDING REMARKS}

In this paper we presented what we believe to be a suitable architecture for the distributed resource managing on CCCs. The option for a detailed modelling, with a computational intelligent agent representing each physical resource was justified and an appropriated coalition formation and negotiation 
protocol for both inter and intra coalition negotiation were presented. The economical options were also justified and an application example that clarifies the different concepts was developed.

As interesting contributions of this work we can point out the multi-agent architecture that demonstrates to be adequate to this class of problems and the negotiation protocol including both inter and intra coalition negotiation. Even in a simple laboratory prototype such as the one we are developing we believe that interesting ideas can be tested and useful solutions for complex problems such as the resource managing in this type of companies can be found.

The next step of our work will be the introduction of learning capabilities on the negotiating agents in order to achieve the dynamical adaptation of the economical parameters to the market conditions at each moment. Another interesting development is the introduction of multi-issue negotiation. The price negotiation as it is actually done is important and typical of the majority of the automatic negotiation system. However, the multi-issue negotiation is much more realistic because it allows the negotiation of different evaluation parameters such as quality, speed, reliability, etc, that are often taken into consideration on real world negotiations.

\section{Acknowledgments}

We would like to thank the reviewers for their helpful comments on an earlier draft of this paper. This work was partially supported by the program PRAXIS XXI under contract TPAR/2037/95.

\section{REFERENCES}

[1] Graphics, Visualization, \& Usability Center's (1997), "CVU Center", <www.gvu.gatech.edu>

[2] Davis, R. and Smith, R.G. 1983. Negotiation as a metaphor for distributed problem solving. In Artificial Intelligence, 20(1), pp. 63-109.

[3] Doorenbos, R., Etzioni, O., and Weld, D., "A scalable comparison-shopping agent for the world wide web," presented at AGENTS'97, Marina del Rey, 1997.

[4] Durfee, E. H., Coordination of Distributed Problem Solvers. Boston, MA: Kluwer Academic Publishers, 1988.

[5] Fisher, K., Kuhn, N., Muller, H. J., and Pishel, M., "Sophisticated and distributed: the transportation domain," in Proceedings of the Fifth European Workshop on Modeling Autonomous Agents and Multi-Agent Worlds (MAAMAW-93). Neuchatel, Switzerland, 1993.
[6] Fonseca, J. M., Oliveira, E. d., and SteigerGarção, A., "Multi-Agent Negotiation Algorithms for Resources Cost Estimation: a case study," in Encontro Português de Inteligência Artificial EPIA'97, vol. 1323, Lecture Notes in Artificial Intelligence. Coimbra, Portugal: Springer-Verlag, 1997, pp. 195-215.

[7] Griffeth, N. D. and Velthuijsen, H., "The negotiating agents approach to run-time feature interaction resolution," in Feature Interactions in Telecommunications Systems, L. G. Bouma and H. Velthuijsen, Eds.: IOS Press, 1994.

[8] Guttman, R. H., Maes, P., Chavez, A., and Dreilinger, D., "Results from a Multi-Agent Electronic Marketplace Experiment,"1998.

[9] Jennings, N. R., Corera, J., Laresgoiti, I., Mamdani, E. H., Perriolat, F., Sharek, P., and Varga, L. Z., Using ARCHON to develop real-world DAI applications for electricity transportation management and particle acceleration control, IEEE Expert, vol. 11, pp. 60-88, 1996.

[10] Jennings, N. R., Faratin, P., Johnson, M. J., Norman, T. J., O'Brien, P., and Wiegand, M. E., Agent-based business process management, Int. Journal of Cooperative Information Systems, vol. 5, pp. 105-130, 1996.

[11] Maes, P., "Agents that reduce work and information overload," in Communications of the ACM, vol. 37, 1994, pp. 31-40.

[12] Oliveira, E. and Shmeil, M. 1995. Detecting the Opportunities of Learning from the Interactions in a Society of Organisations. SBIA-95 XIIth Brazilian Symposium on Artificial Intelligence.

[13] Oliveira, E, Fonseca, J.M. and Steiger-Garçào. Negotiation Between Economically Motivated Agents. Proc. of BASYS'96: 2nd International Conference on Architectures and Design for Balanced Automation Systems, pp. 329-338. Chapman \& Hall.

[14] Oliveira, E., Fonseca, J. M., and Steiger-Garção, A. MACIV - A DAI Based Resource Management System, International Journal on Applied Artificial Intelligence, vol. 11, pp. 525-550, 1997.

[15] Parunak, H. V. D., "Manufacturing experience<with the contract net," in Distributed Artificial Intelligence, M. Huhns, Ed. San Mateo, CA: Pitman Publishing, 1987, pp. 285-310.

[16] Ketchpel, S. 1993. Coalition Formation Among Autonomous Agents. Lecture Notes in Artificial Intelligence 957, pp. 73-88. Springer Verlag.

[17] Kraus, S. Wilkenfeld J., Zlotkin, G. 1995. Multiagent negotiation under time constraints. Artificial Intelligence 75, pp 297-345. Springer Verlag. 
[18] Krulwich, B., "The BargainFinder agent: Comparison price shopping on the Internet," in Bots, and other Internet Beasties, J. Williams, Ed. Indianapolis: Macmillan Computer Publishing, 1996, pp. 257-263.

[19] Kuhn, N., Müller, H. J. and Müller J. P. 1993. Task Decomposition in Dynamic Agent Societies. In Proc. of the Fifth European Workshop on Modelling Autonomous Agents and Multi-Agent Worlds (MAAMAW-93), Neuchatel, Switzerland.

[20] Ljunberg, M. and Lucas, A., "The OASIS air traffic management system," presented at Second Pacific Rim International Conference on AI, Seoul, Korea, 1992.

[21] Rosenschein, J. R. and Zlotkin G. 1994. Rules of Encounter. MIT Press.
[22] Sandholm, T. W. and Lesser, V. R., 1995(a) Issues in automated negotiation and electronic commerce: Extending the contract net framework. In Proc. First International Conference on Multiagent Systems (ICMAS-95), San Francisco, June 1995.

[23] Sandholm, T. and Lesser, V. 1995(b). On Automated Contracting in Multi-enterprise Manufacturing. Proc. Improving Manufacturing Performance in a Distributed Enterprise: Advanced Systems and Tools, Edinburgh, Scotland, pp. 33-42, July 13-14.

[24] Sandholm, T. W. (1996). Negotiation Among Self-Interested Computationally Limited Agents. $\mathrm{PhD}$ Thesys, University of Massachusetts at Amherst. [25] Widerhold G., Genesereth M. The basis for Mediation. Proceedings of COOPIS'95 Conference, Vienna, Austria 1995.

[26] Network Wizards (July 1998), "Internet Domain Survey", http://www.nw.com 